Ann. Génét. Sél. anim., I970, 2 (4), 38г-39г.

\title{
ANATOMIE MICROSCOPIQUE COMPARÉE DU TISSU MUSCULAIRE SQUELETTIQUE DE PORCS LARGE-WHITE ET PIETRAIN
}

\author{
B.L. DUMONT et O. SCHMITT \\ avec la collaboration de Thérèse DEgas, Brigitte Gatebors et Joséphine Roussel. \\ Laboratoire de Recherches sur la Viande, \\ Centre national de Recherches zootechniques, 78-Jouy-en-Josas \\ Institut national de la Recherche agronomique
}

\begin{abstract}
RÉSUMÉ
La structure des muscles demi-membraneux et anconé externe a été comparée chez quatre porcs Large-White et trois Piétrain, de poids de carcasse voisin. On a observé une nette différence, au sein de chaque race, entre les deux muscles et, pour chaque muscle, l'influence de la race ne s'est pas manifestée pareillement. Le muscle demi-membraneux présente, chez le $P i e ́$ train une hypertrophie en masse et en volume qui s'explique par une augmentation du diamètre moyen des fibres musculaires $(88,70 \mu$ contre $78,76 \mu$ ) et de la surface des myoskhènes. Le nombre de faisceaux primaires est semblable dans les deux races. Par contre, le muscle anconé externe, dont les fibres musculaires sont un peu plus réduites, dont les myoskhènes et les faisceaux primaires sont, compte tenu de sa masse, relativement plus nombreux, ne présente pas de caractère d'hypertrophie chez le Piétrain.

L'intérêt de l'analyse microscopique de la section totale du muscle, à des fins génétiques, est discuté. Cette méthode permet non seulement les études comparées du développement musculaire, mais aussi la mise en évidence des fibres musculaires de section arrondie qui paraissent nettement plus abondantes, dans l'un et l'autre muscle, chez le Piétrain que chez le Large-White.
\end{abstract}

\section{INTRODUCTION}

Les études concernant l'anatomie microscopique du tissu musculaire squelettique des animaux domestiques n'ont interessé, jusqu'à maintenant, que quelques caractères des fibres musculaires considérées dans des échantillons de petite taille. On a pu ainsi préciser, pour différents types d'animaux, le diamètre des fibres, leur densité et, plus récemment, leur équipement enzymatique.

L'analyse de tels échantillons (de taille généralement inférieure à cinq millimètres) ne donnait qu'une vue très fragmentaire de la structure du muscle. L'in- 
troduction d'une nouvelle méthode d'exploration reposant sur l'analyse histologique de l'ensemble de la surface de section des muscles (Schmitr et Dumont, I969) permet d'aborder maintenant les études de la structure musculaire en vraie grandeur et de préciser, en particulier, les relations existant entre la nature de la trame du tissu conjonctif et le développement des fibres.

On peut de la sorte caractériser les unités d'organisation, appelées myoskhènes, que paraît révéler l'examen de la trame conjonctive relativement homogène qui sépare certains groupes de faisceaux lorsqu'on examine la coupe d'un muscle à un très faible grossissement. Le présent travail rapporte les résultats obtenus par application de cette technique à la comparaison de muscles de porcs LargeWhite et Piétrain, entre lesquels il existe, pour des sujets de même poids, des différences dans l'importance relative et le volume des muscles (DumoNT et al., I969). Il a été montré que la manifestation de ces caractères chez le Piétrain, qui présentent des analogies avec ceux constatés chez le type " culard " dans l'espèce bovine (LAUVERGNE et al., I963), résulterait d'un déterminisme génctique simple (OLLIVIER et LAUVERGNE, I967), par intervention d'un gène majeur à très forte action potentielle (OLLIVIER, I968) dont les conséquences sur la structure musculaire n'avaient pas encore été explorées.

\section{MATÉRIELS ET MÉTHODES}

On a considéré quatre animaux de race Large-White (3 mâles castrés et une femelle) et trois mâles castrés de race Piétrain dont les principales caractéristiques de carcasse sont indiquées dans le tableau I. Les sujets Large-White qui provenaient des troupeaux de la Station de Recher-

TABLEAU I

Caractéristiques des animaux de l'expérience

\begin{tabular}{|c|c|c|c|c|c|c|c|c|}
\hline \multicolumn{2}{|l|}{ Race } & \multicolumn{3}{|c|}{$\begin{array}{l}\text { Numéro et sexe des } \\
\text { animaux }\end{array}$} & \multirow{2}{*}{$\begin{array}{c}\begin{array}{c}\text { Poids de la } \\
\text { demi-car- } \\
\text { casse } \\
(\mathrm{kg})\end{array} \\
\\
\\
34,4 \\
35, \mathrm{I} \\
35,8 \\
36,5\end{array}$} & \multirow{2}{*}{$\begin{array}{c}\text { Longueur de } \\
\text { la carcasse } \\
\text { (cm) } \\
\\
\\
99,0 \\
98,5 \\
99,0 \\
99,5\end{array}$} & \multirow{2}{*}{$\begin{array}{c}\begin{array}{c}\text { Epaisseur du } \\
\text { lard }\end{array} \\
\text { (mm) } \\
\\
23 \\
22 \\
26 \\
25\end{array}$} & \multirow{2}{*}{$\begin{array}{c}\begin{array}{c}\% \text { de } \\
\text { muscles }\end{array} \\
\\
\\
\\
47,7 \\
49,3 \\
49,2 \\
48,3\end{array}$} \\
\hline Large-White & $\cdot \cdot \cdot$ & $\begin{array}{l}\text { I } \\
2 \\
3 \\
4\end{array}$ & $\begin{array}{l}\text { mâle castré } \\
\text { mâle castré } \\
\text { mâle castré } \\
\text { femelle } .\end{array}$ & \begin{tabular}{ll|}
$\cdot$ & $\cdot$ \\
$\cdot$ & $\cdot$ \\
$\cdot$ & $\cdot$ \\
$\cdot$ & $\cdot$
\end{tabular} & & & & \\
\hline Piétrain. . & $\cdot \cdot \cdot$ & $\begin{array}{l}5 \\
6 \\
7\end{array}$ & $\begin{array}{l}\text { mâle castré } \\
\text { mâle castré } \\
\text { mâle castré }\end{array}$ & \begin{tabular}{ll|}
$\cdot$ & $\cdot$ \\
$\cdot$ & $\cdot$ \\
$\cdot$ & $\cdot$
\end{tabular} & $\begin{array}{l}37,0 \\
34,0 \\
30,1\end{array}$ & $\begin{array}{l}87,0 \\
86,6 \\
87,2\end{array}$ & $\begin{array}{l}26 \\
19 \\
19\end{array}$ & $\begin{array}{l}53,2 \\
65,5 \\
60,8\end{array}$ \\
\hline
\end{tabular}

ches sur l'Élevage des porcs furent sacrifiés aux abattoirs du C.N.R.Z. et, après ressuyage,stockés à $+4^{\circ} \mathrm{C}$. Les sujets Piétrain issus d'élevages privés du Nord de la France furent abattus aux abattoirs des établissements CABY à Saint-André-les-Lille (59) puis, 36 heures après leur sacrifice, transportés dans notre service où ils furent stockés à $+4^{\circ} \mathrm{C}$. Trois jours après l'abattage la moitié gauche des animaux fut pesée, mesurée et disséquée. La longueur de la carcasse était définie par la distance séparant le bord antérieur de la symphyse ischio-pubienne du milieu du bord antérieur de la première côte. L'épaisseur du lard était la moyenne des épaisseurs au niveau du dos (épaisseur minimum) et au niveau du rein (au-dessus du milieu du fessier moyen). 
A l'issue de la dissection quinze muscles furent prélevés aux fins d'études histologiques, parmi lesquels figuraient le muscle demi-membraneux (M. Semi membranosus) et le muscle anconé externe ( $M$. Triceps brachii caput laterale). On a appliqué à ces muscles la technique de préparation précédemment décrite (Scmhiт et Dumont, 1969) en utilisant comme colorant la picrofuchsine de VAN Gieson. On a considéré des coupes de $15 \mu$ d'épaisseur, pratiquées dans la partie médiane des muscles au niveau de leur plus grand développement en volume qui a été apprécié par la prise du contour des muscles au moment de la préparation des échantillons, après congélation rapide et avant la fixation.

Après coloration, en utilisant les coupes comme négatifs, on a obtenu des positifs géants sur papier calibré. Compte tenu de la rétraction présentée par l'échantillon au cours de son traitement histologique, le grandissement linéaire par rapport aux dimensions de l'échantillon frais fut de 10. Après avoir procédé à l'identification des différents myoskhènes, on a évalué leur surface individuelle par découpage des photographies et par pesée. Les faisceaux primaires de fibres musculaires ont été comptés, dans chaque myoskhène, sur une reproduction photographique de même dimension. Le plus grand diamètre des sections de fibres musculaires d'un grand et d'un petit myoskhène pour chacun des muscles a été mesuré. A cet effet on a réalisé un autre type de tirage sur papier à un grandissement linéaire de l'ordre de 130. Enfin à partir de photographies de la surface totale du muscle obtenu à un grandissement linéaire de 20 , on a déterminé le nombre de fibres dites " géantes présentes dans chacun des myoskhènes.

La mesure du diamètre des fibres et la détermination des fibres "géantes " ont été réalisées sur trois animaux dans chaque race (animaux $1,3,4$ dans la race Large-White, animaux 5,6 et 7 dans la race Piétrain).

\section{RÉSULTATS}

Pour chacun des muscles, les 3 principales caractéristiques macro- et microscopiques sont rassemblées dans les tableaux 2 et 3 et sont illustrées dans les figures I et 2.

Les caractères propres aux faisceaux primaires sont représentés dans les figures 3, ainsi que dans les tableaux 4 et 5 .
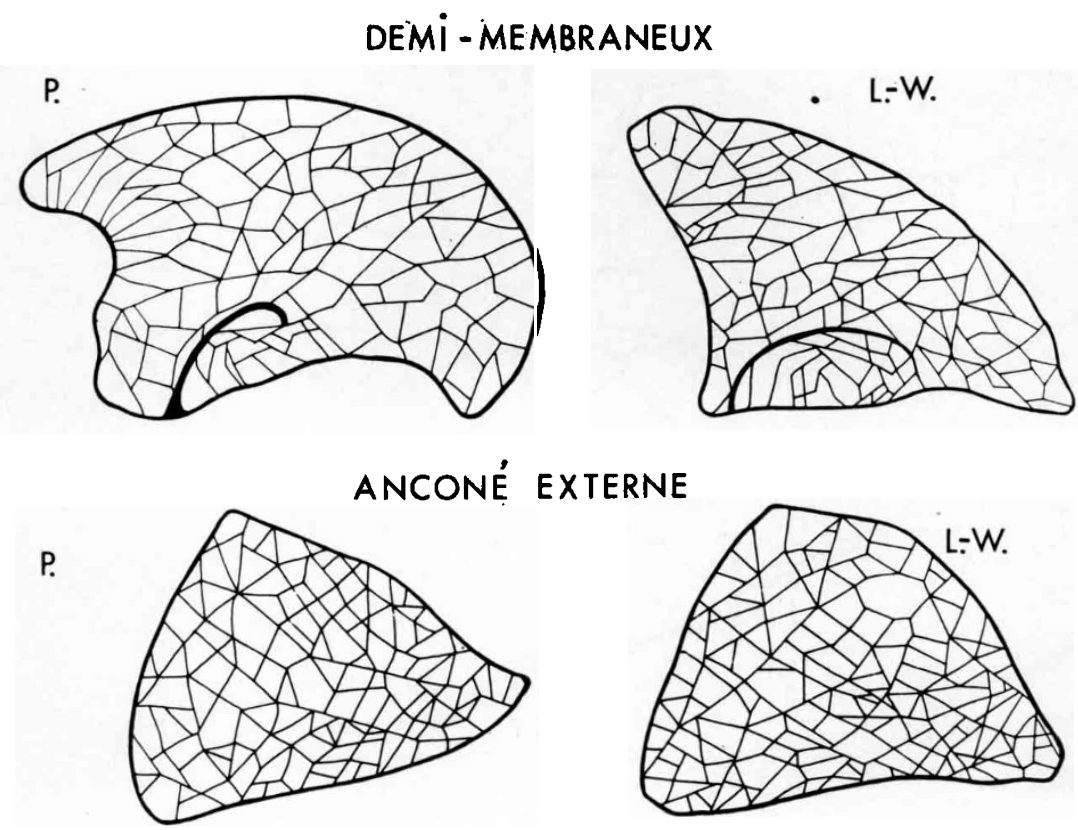

F1G. 1. - Structure schématique de la trame de tissu conjonctif délimitant les myoskhènes chez le Pitérain (P) et le Large-White (L.-W) dans le cas du muscle demi-membraneux $(\times 0,55)$ et du muscle anconé externe $(\times 0,88)$. 

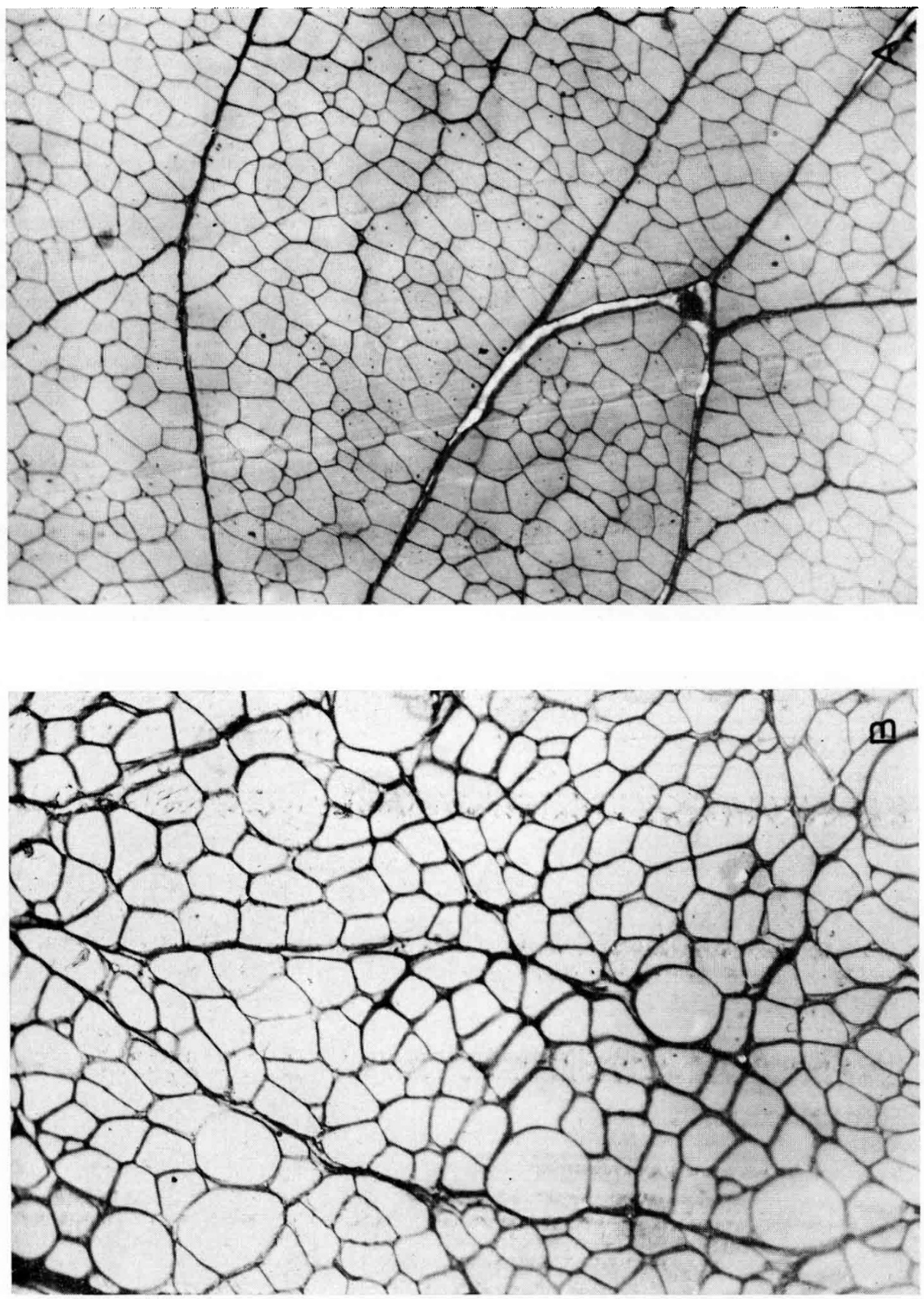

FIG. 2. - Fibres musculaires caractéristiques de porcs Large-White (haut) et Piétrain (bas). 
Les tableaux 6 et 7 indiquent les paramètres concernant les fibres musculaires.

\section{DISCUSSION}

L'examen du tableau 2 fait apparaître que le muscle demi-membraneux est, chez les sujets Piétrain, plus lourd (en valeur absolue et en valeur relative) et plus développé dans sa partie médiane qu'il ne l'est chez les Large-White. Ceci

\section{TABLEAU 2}

Caractéristiques des muscles demi-membraneux

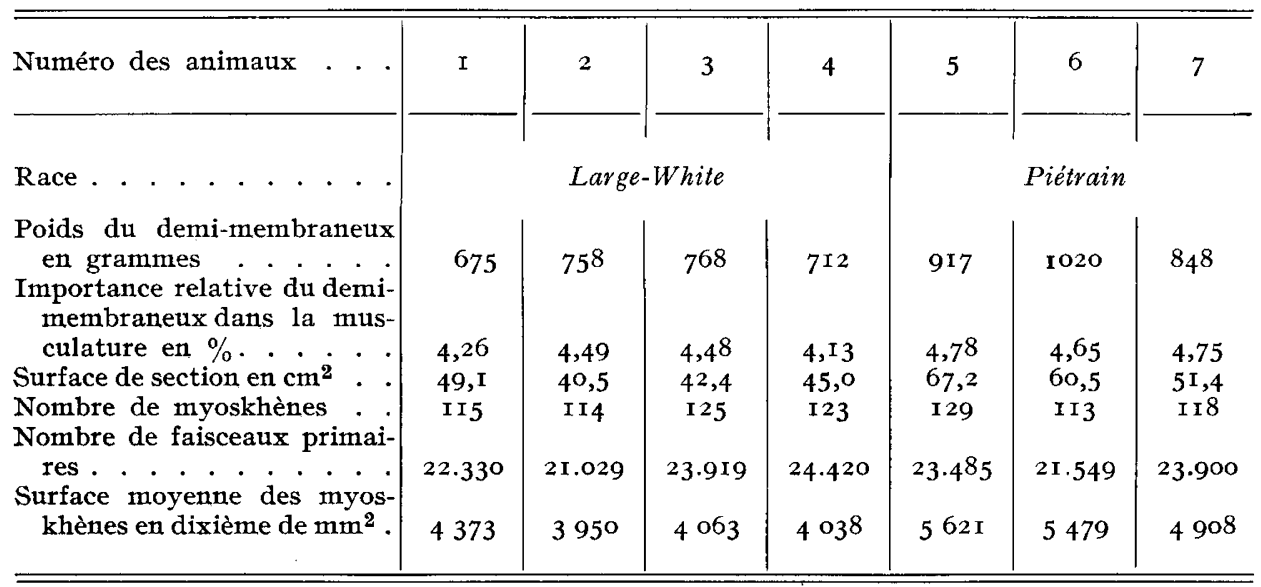

TABLEAU 3

Caractéristiques des muscles anconés externes

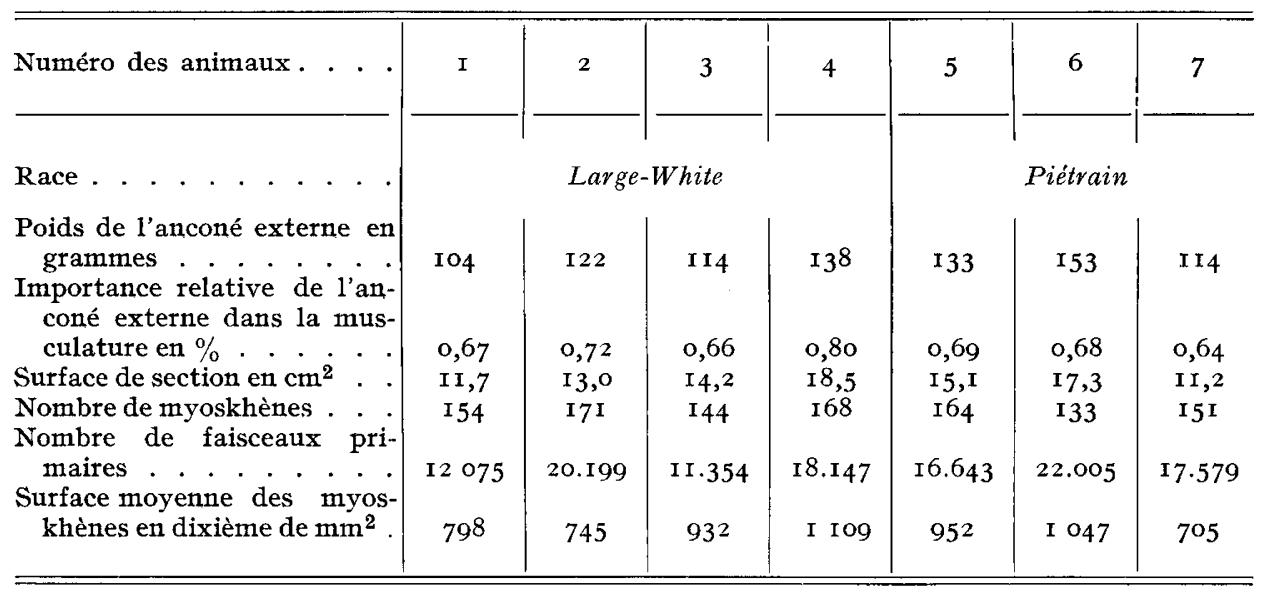


conduit à considérer ce muscle comme étant particulièrement sujet à 1'hypertrophie. A l'inverse, les résultats du tableau 3 montrent que le développement pondéral et volumique du muscle anconé externe est très voisin dans les deux races. L'hypotrophie, chez les Piétrain, du muscle anconé externe n'est d'ailleurs pas exclue.

C'est le caractère opposé dans leur développement musculaire qui nous a conduits à retenir ces deux muscles, en premier lieu, dans l'étude des caractéristiques microscopiques de la musculature des porcins (en tenant compte par ailleurs du fait que nous ne nous intéressons - sur un plan de la valeur technologique des animaux - qu'aux muscles présentant des masses au moins égales à $0,5 \%$ du poids de la musculature).

\section{I. - Muscle demi-membraneux}

Les données $\mathrm{du}$ tableau 2 amènent à conclure qu'indépendamment du caractère d'hypertrophie exprimé par les animaux Piétrain, les paramètres fondamentaux de l'organisation musculaire liés aux faisceaux paraissent équivalents dans les deux races : le nombre des myoskhènes, le nombre de faisceaux primaires, et par suite le nombre moyen de faisceaux primaires par myoskhène sont voisins. Dans la figure 3 , on peut voir aussi que la distribution de fréquence des myoskhènes en fonction du nombre de faisceaux primaires qu'ils renferment est pratiquement la même dans les deux races. Par contre, les caractéristiques liées aux fibres sont très différentes. Malgré leur hétérogénéité dans leur dimension et dans leur forme, les fibres des individus Piétrain ont en moyenne un diamètre supérieur de 1'ordre de Io microns à celle des sujets Large-White. Cette supériorité se traduit, entre autre, par un grossissement des myoskhènes qui, pour un même nombre de faisceaux présentent des surfaces plus grandes (cf. tableau 4). Les coefficients de regression de la surface des unités de structure sur le nombre de faisceaux primaires sont plus élevés chez les Piétrain et représentent une expression du degré

TABLEAU 4

Relations entre la surface des myoskhènes en dixième de $\mathrm{mm}^{2}(\mathrm{Y})$ et le nombre de faisceaux primaires (x) qu'ils renferment

Muscle demi-membraneux

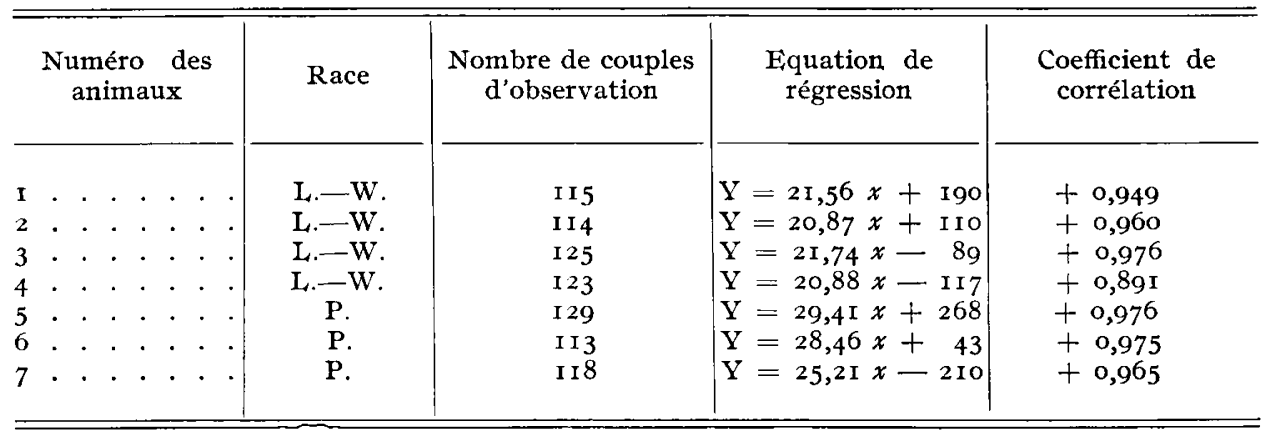


d'hypertrophie du muscle, essentiellement due à l'augmentation de la taille des fibres. En considérant la répartition des myoskhènes en fonction de leur surface (fig. 3) on peut constater que l'intervalle de variation de la surface des myoskhè-

Nbre Myoskh. \%
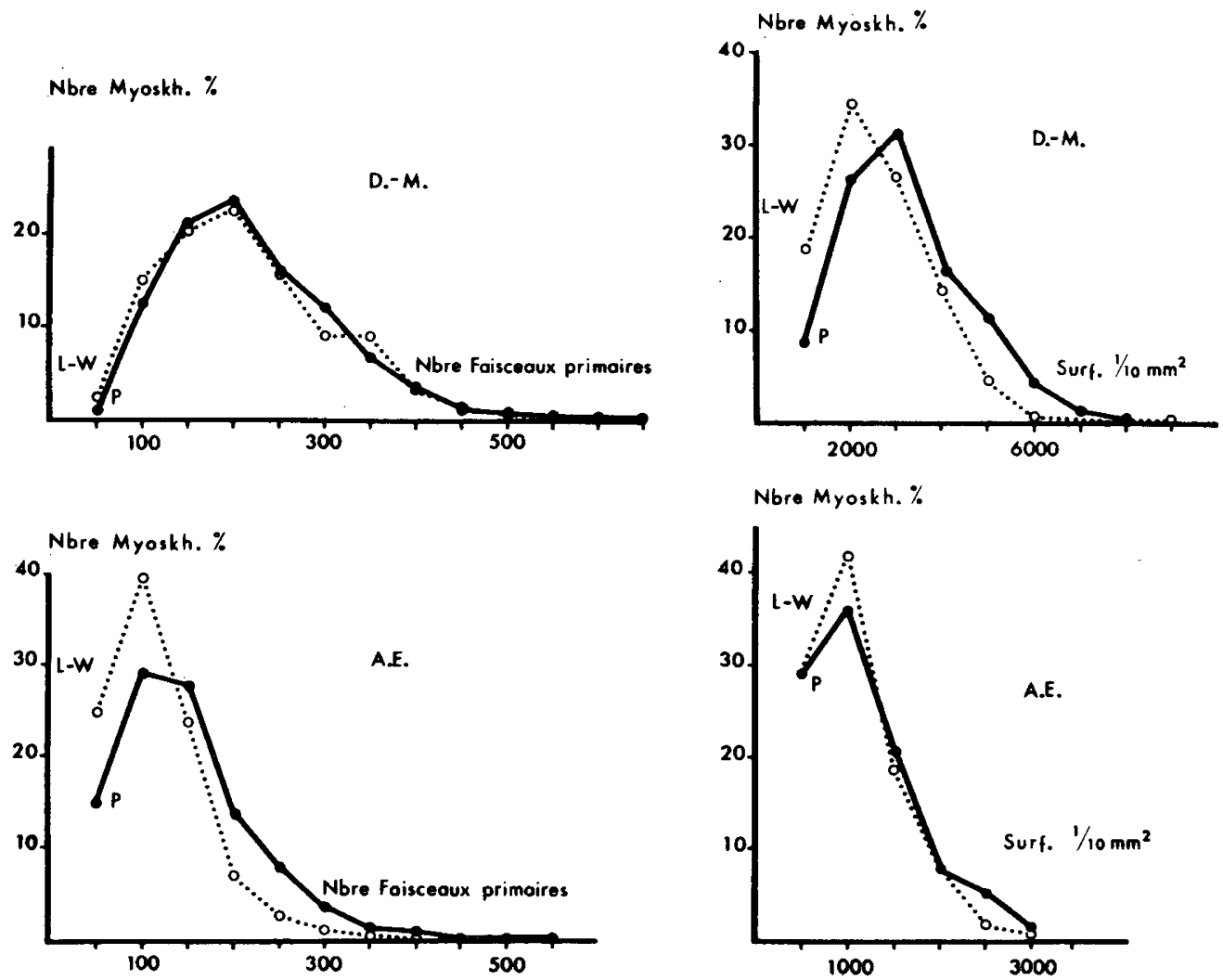

Fig. 3. - Répartition, en pourcentage, des myoskhènes chez le Large-White (L.W.) et le Piétrain (P) en fonction du nombre de faisceaux primaires qu'ils renferment (à gauche), et de leur surface (à droite), dans le cas du muscle demi-membraneux (D.-M.) et du muscle anconé externe (AE).

nes est voisin dans les deux races et relativement important. Toutefois, chez le Piétrain, le pourcentage des grands myoskhènes est plus élevé. Il est à noter que bien que la répartition des myoskhènes de grande et petite section, à l'intérieur du muscle, soit aléatoire (fig. I), les grands myoskhènes sont relativement plus nombreux dans la portion postéro-interne du muscle. Cette zone correspond, précisément, aux directions privilégiées dans laquelle 1'hypertrophie peut se manifester avec le plus d'ampleur (Dumont et al., Ig69).

Il faut signaler d'autre part que l'examen comparé des surfaces de section musculaire totale révèle chez le Piétrain une trame de tissu conjonctif plus fine. Ce caractère est à rapprocher du plus grand développement volumique du muscle demi-membraneux dans cette race. En effet, l'analyse des propriétés de la trame générale du tissu conjonctif en fonction du développement d'un muscle quel- 
conque nous conduit à faire l'hypothèse qu'à l'augmentation de la surface de ses fibres, de ses faisceaux primaires et, par voie de conséquence, de ses myoskhènes, correspond une distension de sa trame conjonctive originelle, conduisant à un amincissement des parois conjonctives qui limitent ses myoskhènes.

Dans le cas du muscle demi-membraneux de Piétrain, pour expliquer la finesse de la trame conjonctive, pourrait s'ajouter à l'effet du plus grand dévelopment musculaire une moindre teneur en tissu conjonctif interne (périmysium) d'origine génétique, comme le laissent supposer les déterminations du taux d'hydroxyproline effectuées par Boccard (I968) sur le muscle entier, avec son epimysium.

TABLEAU 5

Relations entre la surface des myoskhènes en dixième de $\mathrm{mm}^{2}(\mathrm{Y})$ et le nombre de faisceaux primaires (x) qu'ils renferment

Muscle anconé externe

\begin{tabular}{|c|c|c|c|c|c|c|}
\hline & $\begin{array}{l}\text { Numéro des } \\
\text { animaux }\end{array}$ & & Race & $\begin{array}{c}\text { Nombre de couples } \\
\text { d'observation }\end{array}$ & $\begin{array}{l}\text { Equation de } \\
\text { régression }\end{array}$ & $\begin{array}{l}\text { Coefficient de } \\
\text { corrélation }\end{array}$ \\
\hline $\begin{array}{l}1 \\
2 \\
3 \\
4 \\
5 \\
6 \\
7\end{array}$ & 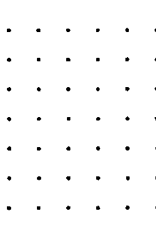 & $\begin{array}{ll}\cdot & \cdot \\
\cdot & \cdot \\
\cdot & \cdot \\
\cdot & \cdot \\
\cdot & \cdot \\
\cdot & \cdot \\
\cdot & \cdot\end{array}$ & $\begin{array}{l}\text { L.-W. } \\
\text { L.-W. } \\
\text { L.-W. } \\
\text { L.-W. } \\
\text { P. } \\
\quad \text { P. } \\
\text { P. }\end{array}$ & $\begin{array}{l}\text { I } 54 \\
\text { I } 7 \text { I } \\
\text { I I } \\
\text { I } 68 \\
\text { I64 } \\
\text { I } 33 \\
\text { I } 5 \text { I }\end{array}$ & $\begin{array}{l}\mathbf{Y}=10,75 x-4 \mathbf{1} \\
\mathbf{Y}=6,28 x-23 \\
\mathbf{Y}=\mathbf{1} \mathbf{1}, 83 x-3 \\
\mathbf{Y}=\mathbf{1 0 , 5 0 x + 3 6} \\
\mathbf{Y}=9,99 x-56 \\
\mathbf{Y}=5,90 x+67 \\
\mathbf{Y}=6,27 x-22\end{array}$ & $\begin{array}{l}+0,969 \\
+0,965 \\
+0,984 \\
+0,994 \\
+0,983 \\
+0,966 \\
+0,946\end{array}$ \\
\hline
\end{tabular}

Ainsi, contrairement aux conclusions de SELIIER et BoccARD (I969), il apparaît que le développement musculaire et la réduction du collagène chez le Piétrain ne sont pas deux phénomènes indépendants.

Le tableau 7 met en évidence l'existence chez le Piétrain d'un très grand nombre de fibres d'un type particulier, appelées " giants fibers " par CASsENS et al. (I969) qui ont décrit d'autre part les propriétés histochimiques.

Anatomiquement, plus que la taille, c'est essentiellement la forme qui permet de les distinguer facilement des autres fibres. Elles présentent en effet un contour curviligne, circulaire ou elliptique qui contraste avec la forme polygonale des autres fibres (fig. 2). Ces fibres sont présentes chez les Large-White en très faible nombre. On a noté entre Io et Ioo fibres environ dans l'ensemble de la section transversale des muscles demi-membraneux de Large-White qui renferment environ entre 800 ooo et I million de fibres musculaires. On peut donc estimer que la fréquence de ces fibres est de l'ordre de I/IO 000 à I/I00 000 . Dans le cas du Piétrain, la fréquence de ces fibres est comprise entre 0,4 et un p. Ioo. On peut supposer que la plus grande fréquence des fibres arrondies (dites "géantes ") constatée chez les sujets Piétrain serait un caractère propre à la race. 


\section{2. - Muscle anconé externe}

Bien que sa masse relative soit assez voisine d'un animal à l'autre (surtout chez le Piétrain), sa masse absolue est très variable et entraîne, parmi les animaux, des variations pour les caractères qui lui sont liés. C'est le cas du nombre de faisceaux primaires. Par contre, le nombre de myoskhènes paraît, comme dans le cas du muscle demi-membraneux, indépendant de la masse et, par suite, présente des valeurs voisines dans les deux races. Ce nombre relativement important en égard à la surtace de section, traduit par rapport au muscle demi-membraneux un plus grand développement de la trame conjonctive, ce qui est en accord avec les résultats de BoccARD (I968) portant sur la détermination de l'hydroxyproline des deux muscles, et une structuration plus prononcée que met en évidence la figure I. Par contre, l'examen histologique de la section montre que la trame de tissu conjonctif est comparable dans les deux races. Cette situation ne devrait normalement pas entraîner des différences aussi sensibles de teneur en hydroxyproline entre race que celles qu'a indiquées BocCARD (Ig68), à moins que la teneur en collagène de la trame colorée par la picrofuchsine soit différente dans les deux races, ou encore que l'importance de l'épimysium dont tiennent compte les déterminations chimiques mais que ne prend pas en considération l'analyse histologique, soit beaucoup moins prononcée chez le Piétrain.

La surface des myoskhènes est étroitement liée au nombre des faisceaux primaires qu'ils renferment. Les distributions des myoskhènes en fonction du nombre des faisceaux qu'ils contiennent sont très différentes dans les deux races (fig. 3), les Large-White possédant plus de myoskhènes contenant peu de faisceaux primaires.

En moyenne les fibres musculaires du Large-White sont un peu plus petites que celles du Piétrain, ce qui conduit, compte tenu des observations précédentes, à postuler que dans le cas de l'anconé externe les Large-White doivent présenter un nombre total de fibres musculaires supérieur à celui des Piétrain.

La présence de fibres dites "géantes" a été constatée chez tous les animaux à l'exception du numéro 5 où aucune fibre de ce type n'a pu être décelée (tableaux 6 et 7$)$.

TABLEAU 6

Caractéristiques des fibres musculaires des porcs Large-White

\begin{tabular}{|c|c|c|c|c|}
\hline \multirow[t]{2}{*}{ Muscle } & \multicolumn{2}{|c|}{ Diamètre des fibres musculaires } & \multicolumn{2}{|c|}{$\begin{array}{c}\text { Nombre de fibres "géantes" par } \\
\mathrm{cm}^{2}\end{array}$} \\
\hline & $\mathrm{N}$ & moyenne & No anim. & \\
\hline Demi-membraneux & $4^{8.598}$ & $7^{8,76 \mu}$ & $\begin{array}{l}\text { I } \\
3 \\
4\end{array}$ & $<_{<\mathrm{I}}^{2}$ \\
\hline Anconé externe. & 25.074 & $73,59:$ & $\begin{array}{l}\text { I } \\
3 \\
4\end{array}$ & $\begin{array}{l}\text { I } \\
2 \\
3\end{array}$ \\
\hline
\end{tabular}


TABLEAU 7

Caractéristiques des fibres musculaires des porcs Piétrain

\begin{tabular}{|c|c|c|c|c|}
\hline \multirow{2}{*}{ Muscle } & \multicolumn{2}{|c|}{ Diamètre des fibres musculaires } & \multicolumn{2}{|c|}{$\underset{\text { Nombre de fibres "géantes" par }}{\text { cnı }^{2}}$} \\
\hline & $\mathrm{N}$ & moyenne & $\mathrm{N}^{\circ}$ anim. & \\
\hline Demi-membraneux & 39437 & $88,70 \mathrm{k}$ & $\begin{array}{l}5 \\
6 \\
7\end{array}$ & $\begin{array}{r}104 \\
\text { I I } 5 \\
8 \mathrm{I}\end{array}$ \\
\hline Anconé externe. & 19847 & $78,80 \ldots$ & $\begin{array}{l}5 \\
6 \\
7\end{array}$ & $\begin{array}{r}0 \\
81 \\
42\end{array}$ \\
\hline
\end{tabular}

\section{CONCLUSIONS}

Moyennant quelques réserves quant à la généralisation des résultats obtenus sur un nombre restreint d'animaux, on peut toutefois raisonnablement conclure:

I. qu'il existe une différence nette, au sein de chaque race, entre les deux muscles considérés;

2. que, pour chaque muscle, l'influence de la race ne se fait pas sentir de la même façon.

A cet égard, il est intéressant de souligner que les différences de structure musculaire observées entre les deux races permettent d'expliquer l'origine des différences relevées lors des études anatomiques, four ce qui est de la manifestation de l'hypertrophie.

L'utilité de l'analyse histologique de la structure musculaire totale dans la caractérisation des types génétiques paraît ainsi démontrée.

Il serait nécessaire, en particulier, de préciser l'influence des facteurs génétiques sur l'apparition, dans les différents types de muscles, de fibres "géantes " dont la liaison avec la sensibilité au "stress " et avec la myopathie exsudative et dépigmentaire a été récemment avancée (CASSENS et al., I969).

Reçu pour publication en juillet 1970.

\section{REMERCIEMENTS}

Nous tenons à remercier Monsieur ZERT, Directeur de l'Institut technique du Porc pour l'aide précieuse qu'il nous a apportée dans l'obtention des animaux Piétrain et les Ets CABY qui nous les ont fournis, d'après des spécifications très précises.

Nous sommes très reconnaissants à MM. Ollivier et OuHAyous des critiques et suggestions qu'ils nous ont faites à la lecture du manuscrit. 


\author{
SUMMARY \\ COMPARATIVE STUDY OF MUSCULAR STRUCTURE \\ IN PIGS OF LARGE WHITE AND PIÉTRAIN BREED
}

The structure of muscular tissue in pigs has been studied by considering Semi membranosus and Triceps brachii caput laterale from four Large-White and three Piétrain, of similar carcass weight. A great difference was found between the two muscles within each breed. The type of breed has not the same effect upon both muscles. In Piétrain pigs Semi membranosus muscle shows an hypertrophy, which is accompanied by increase of the diameter of the muscle fiber $(88,70 \mu$ versus $78,76 \mu)$, in the myoskhène area, though the number of muscular bundles is practically the same as in Large-Whites. On the contrary, Triceps brachii caput laterale shows no sign of hypertrophy.

The interest of comparative studies of muscular structure in the field of genetics for meat selection is discussed. Such studies could make clear not only the relationship between muscle and connective tissue development, but also point out the frequency of round (or " giant *) fibers which seem to be more numerous in the Piétrain than in the Large-White breed.

\title{
RÉFÉRENCES BIBLIOGRAPHIQUES
}

BOCCARD R., I968. Variation de la teneur en hydroxyproline de muscles de Porcs Large-White et Piétrain. Ann. Zootech., 17, 7I-75.

CAssens R.G., CoOper C.C., BRISKEx E., r969. The occurence and histochemical characterization of giant fibers in the muscle of growing and adult animals. Acta Neuropath., 12, 300-304.

Dumont B.L., Schmitr O., Roy G., I969. Développement musculaire comparé de porcs Piétrain et Large-White. Rec. Méd. vét., 145, 937-948.

Lauvergne J.J., Vissac B., Perramon A., I963. Etude du caractere culard. I. Mise au point bibliographique. Ann. Zootech., 12, 133-156.

OLlivier L., LAUVERGNe J.J., r967. Etude du déterminisme héréditaire de l'hypertrophie musculaire du Porc de Piétrain : premiers résultats. Ann. Méd. vét., 111, ro4-rog.

OLlIVIER I.., I968. Etude du déterminisme héréditaire de l'hypertrophie musculaire du Porc de Piétrain. Ann. Zootech., 17, 393-407.

Schmit O., Dumont B.L., 1969. Méthodes d'analyse de la structure musculaire. Ann. Biol. anim. Bioch. Biophys., 9, I 23-1 34 .

Sellier P., Boccard R., r969. Développement musculaire et taux d'hydroxyproline du muscle dans les races porcines Large-White et Piétrain. Ann. Génét. Sél. anim., 1, I79. 